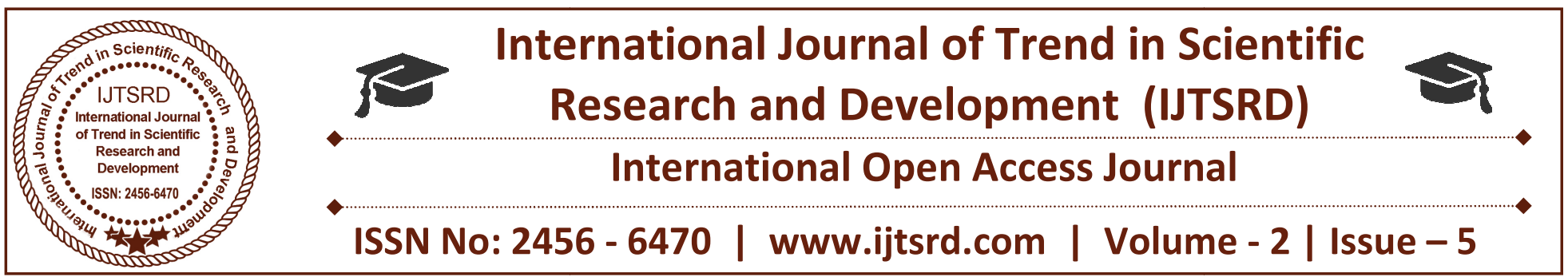

\title{
Fruit Classification and Calories Measurement System
}

\author{
May Zin Oo*, Dr. Mya Thandar Kyu \\ *Assistant Lecturer \\ Department of Electronic Engineering, Pyay Technological University, \\ Republic of the Unoin Myanmar, Pyay Western (Bago), Myanmar
}

\section{ABSTRACT}

Now a day, people around the world are becoming more sensitive to their diet. Fruits contribute to an essential part of the diet because they are a major source of energy, vitamins, fiber, plant chemicals and nutrients. Fruits are naturally low in fat, sodium \& calories and rich in potassium and fiber, vitamin $\mathrm{C}$. The proposed system gives quickly how much calories present in their diet or fruit intake that can be very useful to maintain health without expert dietitian advice. The system will take the images of fruit and using image processing, segmentation and classification it calculates the calorie content in the fruit. This work proposes an algorithm for fruit recognition and its calorie measurement based on the image features such as shape, color and texture. For shape based feature extraction the geometrical region parameters like area, major axis and minor axis are calculated. For color based segmentation the K-Mean Clustering method is used. The gray level cooccurrence matrix (GLCM) is used to calculate different texture features. The user just takes a picture of the fruit image then to recognize the image to detect the type of fruit portion and classify using support vector machine. After classifying the type of the fruit, the calorie of each fruit with generally for $100 \mathrm{~g}$ are derived using standard calories table.

Keyword: Talent Screening, Talent Detection, Talent Development, Talent Selection.

\section{INTRODUCTION}

Obesity is one of the public health challenges our nation is facing today. Obesity rates have grown to epidemic proportions that threaten our economic and national security in the past two decades. Caloric and nutrition imbalance are the result of overweight and obesity at the most basic level. Healthy eating is central to overall health and reduces the risk of nutrition-related chronic diseases and obesity. Obese people are more likely to have serious health condition such as hypertension, heart attack, diabetes, breast and colon cancer and breathing disorder. Imbalance between the amount of food intake. So, in order to lose weight in a healthy way, as well as to maintain a healthy weight for normal people, the daily food intake must be measured [5].

Now a day people across the globe are becoming more interested in watching their weight, eating more healthily, and avoid obesity, a system that can measure calories and nutrition in every day meals can be very useful. Individuals care about the types of fruit they are eating and the nutrients it contains because eating fruits and vegetables is an essential part of leading a healthy diet. Fruits are a major source of energy, vitamins, fiber, plant chemicals and nutrients. They contribute to an essential part of our diet. Fruits are naturally low in fat, sodium \& calories and rich in potassium and fiber, vitamin C. A diet high in fruit can help human against cancer, diabetes, heart diseases etc.

Along with health benefits, eating fruits can make weight management easier, according to centers of disease control and prevention (CDCP) [4]. Most produce is in low calories compared to other food. So filling up on these fruit can aid in weight loss or health management. If a sample fruit or a plate of fruit is put in front of human being then he/she will unable to predict the exact or near about calorie count by just observing at it or even inspecting it by hand, because it is impossible to know the exact amount of calories 
so to overcome this problem this system is proposed. Researcher has combined different features like color and texture together in order to recognize fruit more accurately [1].

In this research work, fruit recognition is proposed and its calorie measurement system based on laptop device equipped with a camera. Using image segmentation methods, the fruit portion area with other geometrical parameters and texture features will be extracted from the fruit image. After that, the multi class support vector machine (SVM) technique will classify and identify the type of fruit. This will allow the system to extract the features in an exclusive approach that will give the ability to calculate the calorie of the fruit with the help of nutrient fact table from USDA national nutrient database [3].

In this research, the goal is to train a model that detects all kinds of fruits but due to the extensive computational requirements, in this work limited to the images of five types of fruits apple, avocado, mango, strawberry and banana. An automatic fruit recognition system could serve as a calorie counter for people who are trying to lose weight. People could take a picture of the fruit that they are eating and see the number of calories it contains.

\section{RELATED WORK}

Two relevant existing systems that analyze and recognize fruits using shape-based and texture-based analysis method have been reviewed recognition with the help of image processing technique. The existing systems, fruits shape variation analyzer, fruits recognition and "localization system and tree fruit recognition using texture properties and color data", are presented. Fruits Shape Variation Analyzer for tomato and other plant species has been developed to analyze shape and size of tomato fruit and other plant species [3], such as Butternut squash, yellow squash, large jalapeno, banana pepper, chili pepper, grape, strawberry and Bartlett pear. It can accurately determine boundaries of fruit with different color.

The Analyzer can perform detection for tomato fruit shape variation and describe any other two dimensional fruit shape. It provides intuitive descriptors and output that facilitates the analysis of fruit morphology. They propose an algorithm for fruit classification based on the color and texture features. Mathematical statistics is calculated for each feature and give to artificial neural network (ANN) for classification [4]. Woo Chaw Seng and Seyed Hadi Mirisaee developed a new fruit recognition system, which combines features like size, color and shape. With the help of nearest neighbours classification method fruit images have classified and recognize and show fruit name and user description [5].

The analyzer contains function for manually adjustment the distal and proximal ends of the fruit. Therefore, no matter fruit are positioned at an angle, the analyzer also can correctly identify the distal and proximal ends of the fruit. This method accuracy is different based on the fruit image but the overall is around $80 \%$ to $85 \%[6]$. On tree fruit recognition using texture properties and color data[7], a vision based algorithm presents to locate apples in a single image. Texture based edge detection has been combined with redness measures, and area thresholding followed by circle fitting, to determine the location of apples in the image plane. It was shown that redness works for red apples as well as green apples. This increased texture contrast helped to identify apples separately from background. The algorithm worked equally well for close ups as well as distant images of apples. Results show that the accuracy of system is around $90 \%$.

\section{SYSTEM DESCRIPTION}

The proposed fruit calorie and nutrition measurement system consists of two stages: classification and second is calorie measurement. The block diagram of proposed system shown in Figure 1 gives an idea of system. In the following more about image acquisition, pre-processing, segmentation, feature extraction. classification and calorie measurement steps are discussed.

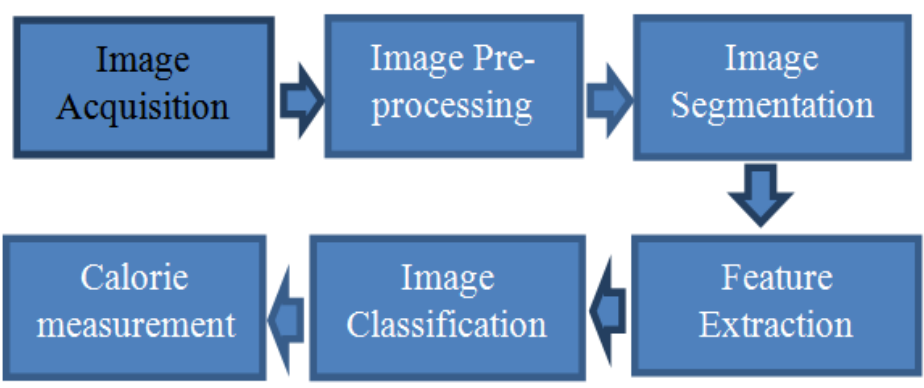

Figure1. Block Diagram of the Proposed System

Five categories of fruit images are captured using webcam and the images acquired were 1280 x 720 pixels in size or more. Few sample databases of fruit images have been collected from fruit market. No special camera set up is required to capture pictures because system will produce output of any picture taken without consideration of special set-up. 
In the Pre-processing step, a simple conversion must be performed on the image to change the image size into a standard format for precise results for system segmentation. Pre-processing steps such as RGB to $\mathrm{L}^{*} \mathrm{a} * \mathrm{~b}$ color conversion, filtering, resizing to $256 \mathrm{x}$ 256 are carried out. The size of each image will be compared with standard size categories. One size category has defined as a standard, which are $256 \mathrm{x}$ 256 pixels for simplicity. Larger images will be reduced to this size before accomplishment of any image-processing technique.

The segmentation phase starts immediately after analysing the pre-processing step. The RGB to $\mathrm{L}^{*} \mathrm{a} * \mathrm{~b}$ color conversion is used for the purpose of interested area (fruit area) segmentation. Convert Image from RGB Color Space to $\mathrm{L}^{*} \mathrm{a} * \mathrm{~b} *$ Color Space. The $\mathrm{L}^{*} \mathrm{a} \mathrm{b}^{*}$ space consists of a luminosity layer ' $\mathrm{L} *$ ', chromaticity-layer ' $a *$ ' and ' $b *$ '. All of the color information is in the ' $a *$ ' and ' $b$ '' layers. After color conversion kmeans clustering is used to classify the colors in $\mathrm{a}^{*} \mathrm{~b}^{*}$ color space. Since the image has 2 colors create 2 clusters.

This research work operates with three different features: colour, texture, and shape, on which are mainly focused in this work. For shape based feature extraction the geometrical region parameters like area, major axis and minor axis are calculated. For color based features Color Coherence Vector (CCV) is used. A color coherence vector (CCV) stores the number of coherent versus incoherent pixels with each color. By separating coherent pixels from incoherent pixels, ccv's provide finer distinctions than color histograms. It describes colours in terms of their shades and brightness. The texture feature plays important role in recognition phase. Texture is calculated by objects physical properties. The texture feature helps in surface determination and shape determination. The gray level co-occurrence matrix (GLCM) and Local ternary patterns (LTP) are used to calculate different texture features. There are three steps to be followed for gray level co-occurrence matrix execution i.e. creating GLCM, specifying the offsets, and deriving statistics from a GLCM. The values of contrast, correlation and energy are considered and are fed to feature vector.

Classification with the Support Vector Machine has been done. The extracted features before revealed will be fed into the SVM classifier so that the classifier returns the fruit name and its calorie value as its output.

The main objective of the proposed system is to estimate the amount of calories and nutrition values for fruit from an image. Thus, caloric estimation is the main, final stage for the system. In fact, the importance of using the already stored nutrient fact tables will also appear in this stage. In general, the system starts to calculate the calories by comparing the inputs from the image with the inputs from the nutrient tables (and calorie amount, which is measured by calories), which are already stored in the application's database. A novel method to measure and estimate the amount of calories in any image is proposed. As mentioned before, this method depends on the association between the standard stored variables in the nutrient fact tables such as calories, and fruit weight with the known variables extracted from the image to calculate.

\section{DESIGN OF THE PROPOSED SYSTEM}

Fruit classification calories measurement system is implemented in MATLAB environment. The proposed system can classify five types of fruits such as apple, avocado, mango, orange and strawberry. Fruit images are JPEG format captured by digital camera or webcam and 1-3 feet distance. And, fruit images are taken from the white background or plain background at day time or night.

The proposed system has two main phases: segmentation and classification. The segmentation phase is composed of load image, k-mean clustering and interesting fruit area extraction. After segmentation GLCM features, shape features, LTP features and $\mathrm{CCV}$ features are extracted. SVM classifier is used for fruit type classify. The flowchart is shown step by step design procedures of the proposed system.

Initially, fruit images are loaded as input into the prooposed system by clicking the Load Image and then selected desired image from open dialogue box. In segmentatoin, the image is converted from RGB color space to $\mathrm{L}^{*} \mathrm{a}$ *b color space because RGB color space has a higher sensitivity to light intensity. Then, color based segmentation is used to obtain the possible region of fruit using kmean clustering. The value of $\mathrm{K}$ can be defined 2-5 range depending on the input image. Therefore, Segment Image button 
includes RGB to $\mathrm{L}^{*} \mathrm{a} * \mathrm{~b}$ conversion and k-mean clustering to get the region of interests. The result image of $\mathrm{k}$-mean clustering based segmentation is shown in the following Figures.

After the fruit area is segmented, the features are extracted. The types of features can be chosen by checking the check box. The combination of color, texture and shape features gives the best classification result than separately used.

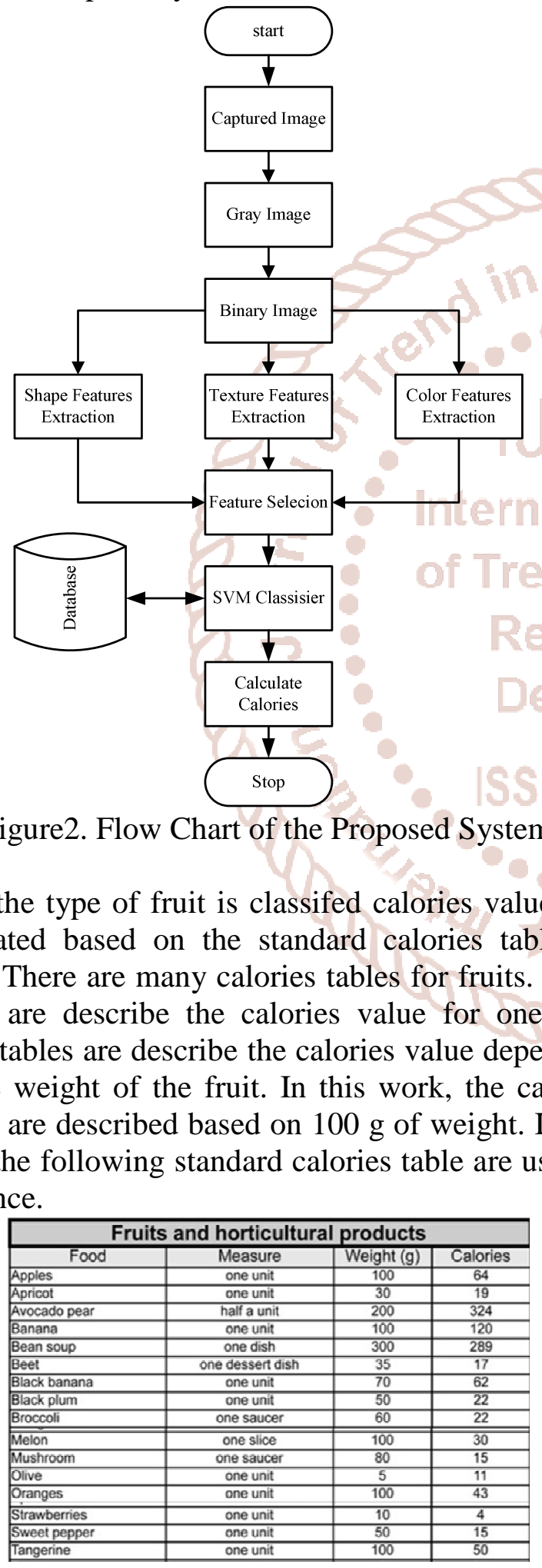

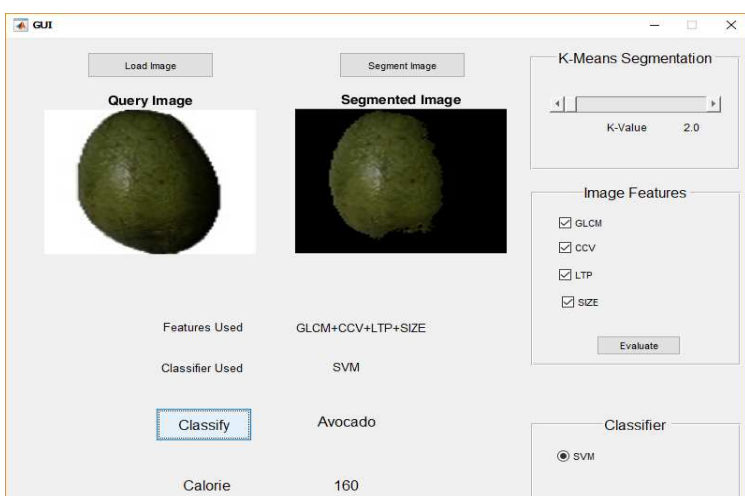

Figure3. Result Image for Classification (Avocado)

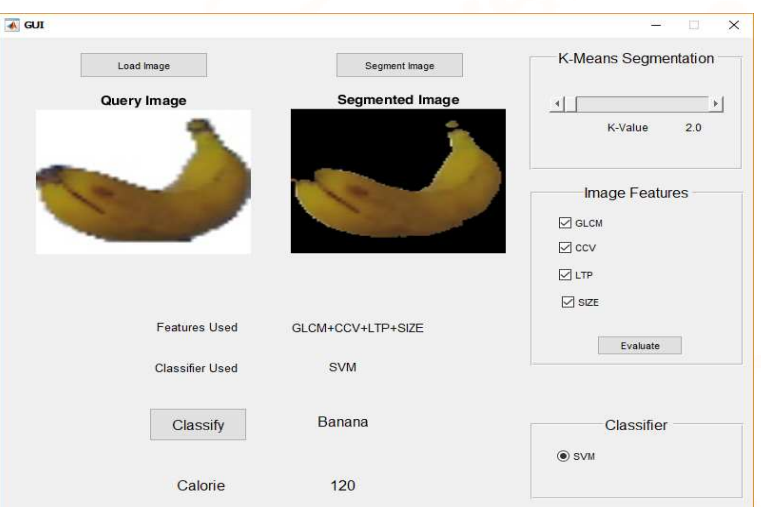

Figure4. Result Image for Classification (Banana)

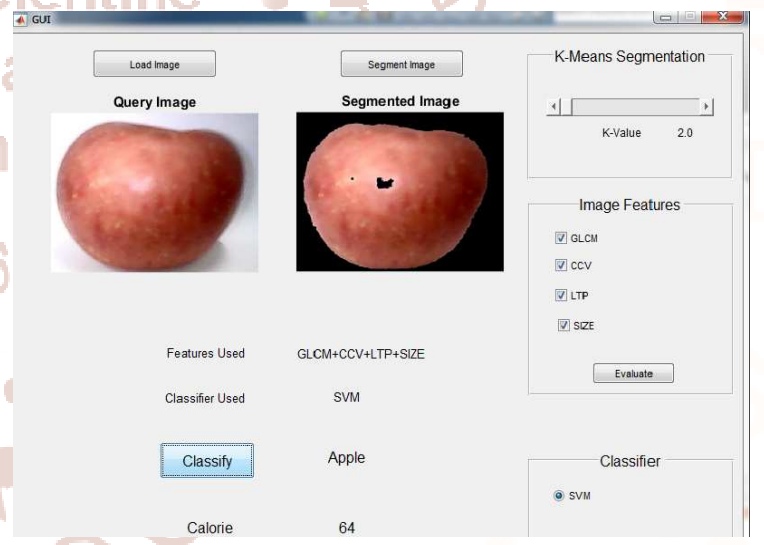

Figure5. Result Image for Classification (Apple)

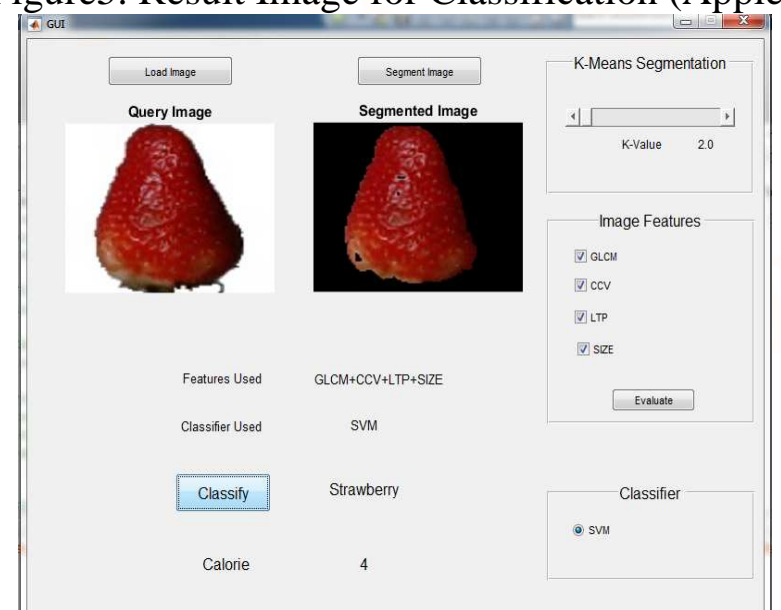

Figure 6. Result Image for Classification (Strawberry) 


\section{PERFORMANCE EVALUATION}

For the best recognition system, recognition accuracy should be high. It is calculated as ratio of number of correctly recognized fruit samples upon total number of samples used in testing. The performance for the system is evaluated using five categories of fruit whose samples are not in dataset. The samples are tested with our system. The proposed system is implemented for testing 20 images for each type of fruits. The 160 images for each type of fruit are used to train in the database.

The result of recognition rate is calculated with the help of equation 4 and the correctly recognised samples and the samples which are not recognised are shown by values in the table1.

Table1. Recognition Rate

\begin{tabular}{|c|c|c|c|c|c|}
\hline $\begin{array}{c}\text { Sr. } \\
\text { No. }\end{array}$ & Fruit Name & $\begin{array}{c}\text { Total } \\
\text { Samples }\end{array}$ & $\begin{array}{c}\text { No. of Fruit } \\
\text { Recognized }\end{array}$ & $\begin{array}{c}\text { Not } \\
\text { Recognized }\end{array}$ & $\begin{array}{c}\text { RR } \\
(\%)\end{array}$ \\
\hline 1 & Apple & 20 & 19 & 1 & 95 \\
\hline 2 & Avocado & 20 & 20 & 0 & 100 \\
\hline 3 & Banana & 20 & 20 & 0 & 100 \\
\hline 4 & Orange & 20 & 19 & 1 & 95 \\
\hline 5 & Strawberry & 20 & 18 & 2 & 90 \\
\hline
\end{tabular}

\section{CONCLUSION}

In this research work, a fruit recognition system that classifies the type of fruit from an image by extracting the color and texture features of the fruit portions from the image is proposed. To reach our goal, we used phone camera or web cam. The designed system is robustness in nature, execution time is approximately 35 seconds. The SVM algorithm is the appropriate and effective classification algorithm to be used in the Fruits Recognition System. The recognition system that has been developed is able to recognize all the test fruit images which are being selected by user or system tester from the fruit selection menu on the system. The recognition results of the system are accurate up to $95 \%$ as mention in above section. The system has ease of use to the user as it has the facility of automatic and manual measurement method. Accuracy of the system is up to the mark. We have successfully implemented a robust system for the correct identification of the fruit and calories measurement.

\section{REFERENCES}

1. Richard O. Duda, P. E. H., David G. Stork, Pattern classification. Second ed. 2001, New York: Wiley.

2. W. K. Jung, I.C.N., Relevance Feedback in Content- Based Image Retrieval System by Selective Region Growing in the Feature Space. Signal Processing: Image Communication, 2003. 18: p. 13.
3. Marin Talbot Brewer, L. L., Kikuo Fujimura, Nancy Dujmovic, Simon Gray, Esther van der Knaap, Development of a Controlled Vocabulary and Software Application to Analyze Fruit Shape Variation in Tomato and Other Plant Species. Plant Physiology, 2006. 141: p. 15-25.

4. Woo Chaw Seng and Seyed Hadi Mirisaee, "A New Method for Fruits Recognition System," 2002.

5. Parisa Pouladzadeh, Shervin Shirmohammadi, and Rana Almaghrabi, "Measuring Calorie and Nutrition from Food Image," In IEEE 2012.

6. A. R. Jimenez, R.C., and J. L. Pons, A survey of Computer Vision Methods for Locating Fruit on Trees. ASAE, 2000. 43: p. 1911-1920.

7. Zhao, J. T., J. Katupitiya, J. , On-tree fruit recognition using texture properties and color data, in international conference on Intelligent Robots and Systems. 2005, IEEE: Edmonton Canada. p. 263-268.

8. Neelamegam, P. Abirami, S. Vishnu Priya, K., \& Rubalya Valantina, S. "Analysis of rice granules using image processing and neural network", In IEEE Conference on Information \& Communication Technologies (ICT), 879-884, 2013.

9. Pouladzadeh, P. Shirmohammadi, S., \& Arici, T., "Intelligent SVM based food intake measurement system", In IEEE International Conference on 
International Journal of Trend in Scientific Research and Development (IJTSRD) ISSN: 2456-6470

Computational Intelligence and Virtual Environments for Measurement Systems and Applications (CIVEMSA), pp.87-92, 2013.

10. Abdulhamid Haidar, Haiwei Dong and Nikolaos Mavridis, "Image Based Date Fruit Classification", IEEE Conference on Ultra modern Telecommunications and Systems, pp. $369-375$, 2015.

11. Maruyama, Y., de Silva, G. C., Yamasaki, T., \& Aizawa, K., "Personalization of food image analysis", In 16th International Conference on Virtual Systems and Multimedia (VSMM), pp.75-
78, 2010. Abdulhamid Haidar, Haiwei Dong and Nikolaos Mavridis, "Image Based Date Fruit Classification", IEEE Conference on Ultra modern Telecommunications and Systems, pp. $369-375$, 2015.

12. Manpreetkour Basantsingh Sardar1 \&. Sayyad D. Ajij, "Fruit Recognition and its Calore Measurement. An Image Processing Approch ," In Journal of Emerging Trends in Computing and Information Sciences 2016.

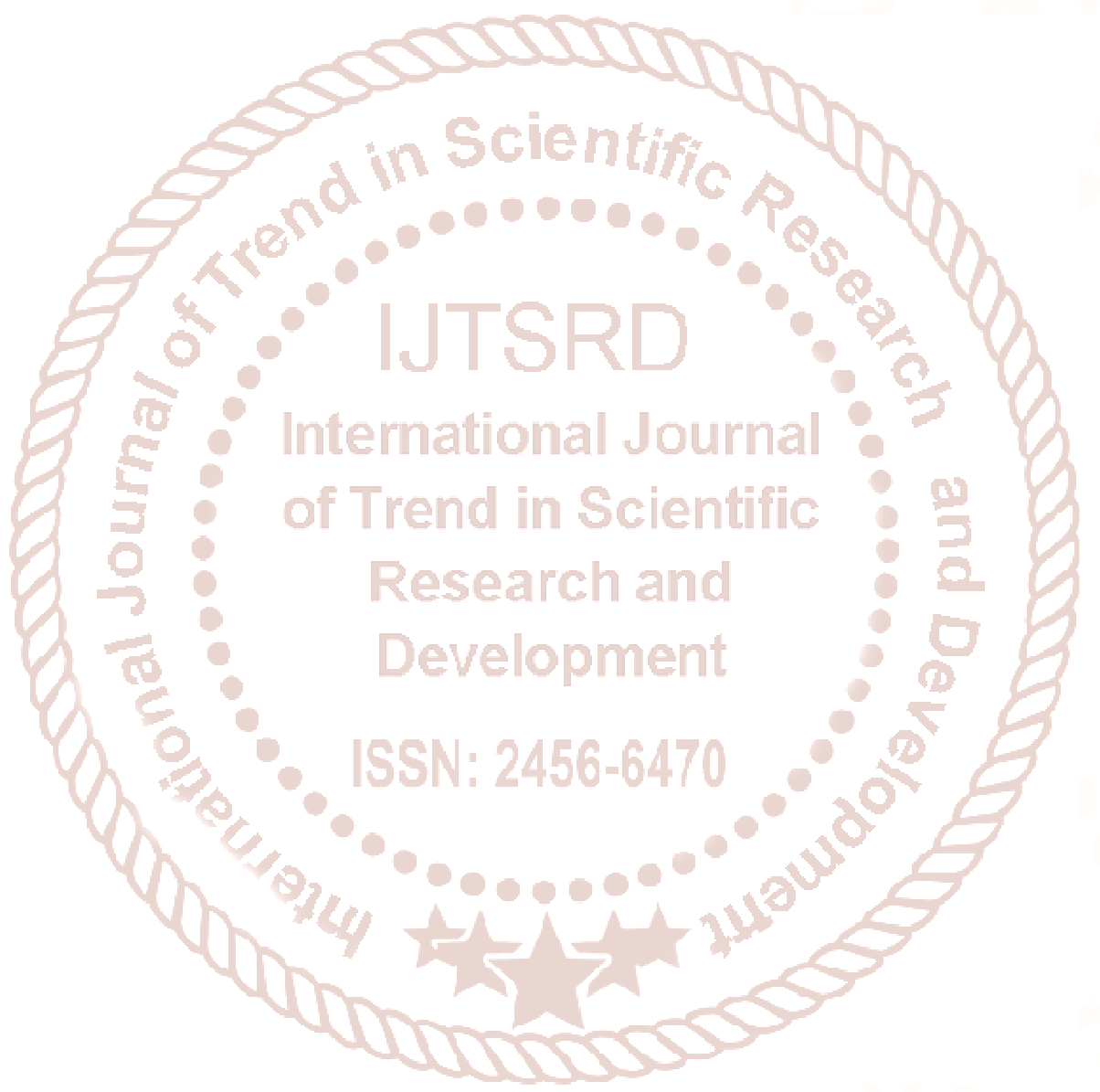

